\title{
Modelo experimental para o estudo da hérnia do disco intervertebral ${ }^{*}$
}

\author{
Experimental model to study intervertebral disc herniation
}

\author{
André luiz de Souza Grava ${ }^{1}$, luiz Fernando Ferrari ${ }^{2}$, Carlos Amílcar Parada ${ }^{3}$, Helton L.A. Defino ${ }^{4}$
}

\section{RESUMO}

Objetivo: Apresentar um modelo experimental de hérnia de disco e sua validação para estudo da hiperalgesia mecânica e térmica produzidas pelo contato do núcleo pulposo (NP) com as estruturas nervosas envolvidas nessa afecção. Métodos: Foram utilizados ratos Wistar, sendo o NP autólogo retirado da região sacrococcígea e depositado sobre a dura-máter, raiz nervosa ou gânglios das raízes dor-

* Trabalho realizado no Laboratório de Bioengenharia do Departamento de Biomecânica, Medicina e Reabilitação do Aparelho Locomotor da Faculdade de Medicina de Ribeirão Preto da Universidade de São Paulo - USP - Ribeirão Preto (SP), Brasil.

1. Pós-Graduando do Departamento de Biomecânica, Medicina e Reabilitação do Aparelho Locomotor da Faculdade de Medicina de Ribeirão Preto da Universidade de São Paulo - USP - Ribeirão Preto (SP), Brasil.

2. Pós-Graduando - Departamento de Farmacologia da Faculdade de Medicina de Ribeirão Preto da Universidade de São Paulo USP - Ribeirão Preto (SP), Brasil.

3. Doutor, Professor Orientador do Departamento de Farmacologia da Faculdade de Medicina de Ribeirão Preto da Universidade de São Paulo - USP - Ribeirão Preto (SP), Brasil.

4. Professor Titular do Departamento de Biomecânica, Medicina e Reabilitação do Aparelho Locomotor da Faculdade de Medicina de Ribeirão Preto da Universidade de São Paulo - USP - Ribeirão Preto (SP), Brasil.

Endereço para correspondência: Helton L.A. Defino, Departamento de Biomecânica, Medicina e Reabilitação do Aparelho Locomotor da Faculdade de Medicina de Ribeirão Preto da USP, Av. Bandeirantes, 3.900, 11o andar - 14048-900 - Ribeirão Preto, SP. Tels.: (16) 3602-2513/3633-7559 - Fax: (16) 3633-0336.

E-mail: hladefin@fmrp.usp.br

Recebido em 22/2/08. Aprovado para publicação em 8/4/08.

Copyright RBO2008 sais L4, L5 ou L6. Os experimentos foram divididos em quatro etapas: 1a) determinação da estrutura nervosa mais sensível ao contato com o NP; 2a) identificação do melhor nível lombar para a indução da hiperalgesia; $3^{\text {a) }}$ ) determinação da ausência de lesão motora; e 4⿳a) determinação da influência do procedimento cirúrgico no desenvolvimento do processo inflamatório. A hiperalgesia foi avaliada nos testes de von Frey eletrônico e de Hargreaves e a função motora, pelo teste de rota-rod. Resultados: O NP induziu hiperalgesia de maior intensidade na pata quando em contato com o gânglio da raiz dorsal (GRD) do que em contato com a dura-máter ou a raiz nervosa. Quando em contato com o GRD-L5, o NP induziu hiperalgesia ainda maior que a induzida pelo contato com os GRDS L4 e L6. Não foram observadas lesão motora e influência do processo inflamatório cirúrgico sobre a hiperalgesia. Conclusão: O GRD é a estrutura mais sensível aos componentes do NP para a produção da hiperalgesia, sendo o quinto nível lombar o que apresentou maior alteração nas sensibilidades mecânica e térmica avaliadas na pata dos animais, de acordo com os métodos utilizados.

$\begin{aligned} \text { Descritores - } & \text { Coluna vertebral; Disco intervertebral; Dor } \\ & \text { lombar; Hiperalgesia; Deslocamento do dis- } \\ & \text { co intervertebral; Ratos Wistar }\end{aligned}$

\section{ABSTRACT}

Objective: The purpose of this study is to present an experimental model of disc herniation and to validate such model to study mechanic and thermal 
hyperalgesia produced by the contact of the nucleus pulposus (NP) with nerve structures involved in this condition. Methods: The authors used Wistar rats, the autologous NP being removed from the sacrococcygeal region and deposited on the dura mater, nerve root, or L4, L5, or L6 dorsal root ganglia. The experiments were divided into four steps: 1) determining the nerve structure that is the most sensitive to the contact with NP; 2) identifying the best lumbar level to induce hyperalgesia; 3) determining absence of a motor lesion; and 4) determining the impact of the surgical procedure upon the inflammatory process. Hyperalgesia was evaluated by the von Frey electronic test and the Hargreaves test, and the motor function was evaluated by the rota-rod test. Results: NP induced higher intensity hyperalgesia in the paw when it was in contact with the dorsal root ganglion (GRD) than when it was in contact with the dura mater or the nerve root. Contact with GRD-L5, led NP to induce even higher hyperalgesia than that induced in the contact with L4 and L6 GRDS. No motor lesion and impact of the surgical inflammatory process on hyperalgesia were observed. Conclusion: GRD is the structure that is most sensitive to NP components to produce hyperalgesia, the fifth lumbar level being that showed the greatest change in the mechanic and thermal sensitivities evaluated in the paws of the animals, under the methods used.

Keywords - Spine; Intervertebral disk; Low back pain;

Hyperalgesia; Intervertebral disk

displacement; Ratos Wistar

\section{INTRODUÇÃO}

O quadro clínico da hérnia do disco intervertebral foi descrito por Mixter há mais de $70 \operatorname{anos}^{(1)}$. Entretanto, os mecanismos fisiopatológicos básicos participantes da gênese dos sintomas relacionados com as raízes nervosas envolvidas nessa doença ainda não estão totalmente esclarecidos.

A manifestação clínica da hérnia de disco lombar está diretamente relacionada com as alterações fisiopatológicas dos nervos das regiões lombar e sacral.
Sua expressão clínica pode ser dor e disfunção da raiz nervosa afetada, sendo as principais características: a dor irradiada no trajeto nervoso acometido, disfunção nervosa, alterações motoras, sensitivas e dos reflexos relacionados com a respectiva raiz $^{(1-4)}$.

Os estudos realizados indicam que os sintomas da hérnia de disco lombar estão associados com o fator mecânico da compressão da raiz nervosa pelo fragmento do disco intervertebral herniado e pela atividade biológica e bioquímica dos componentes do disco intervertebral que entram em contato com o tecido nervoso $^{(4-8)}$.

A participação desses componentes do disco intervertebral na indução da dor da raiz nervosa tem sido clínica e experimentalmente demonstrada, tendo sido observado que a presença do núcleo pulposo torna o tecido nervoso sensível à deformação mecânica ${ }^{(4-9)}$. A avaliação da dor nos modelos experimentais disponíveis é mais difícil e tem sido baseada na avaliação da resposta comportamental a estímulos térmicos e mecânicos de alta intensidade ${ }^{(10)}$.

O objetivo do presente trabalho foi o desenvolvimento de um modelo experimental para estudo das alterações nervosas provocadas pelos componentes do disco intervertebral, na ausência de compressão mecânica da raiz nervosa e com ênfase no aumento da sensibilidade à dor.

\section{MÉTODOS}

Foram utilizados ratos Wistar machos, pesando entre $220 \mathrm{~g}$ e $250 \mathrm{~g}$, fornecidos pelo Biotério Central da Faculdade de Medicina de Ribeirão Preto-USP. Os procedimentos cirúrgicos e os testes comportamentais foram realizados no período das $8: 00 \mathrm{~h}$ às $17: 00 \mathrm{~h}$ Os experimentos foram realizados de acordo com as Normas Internacionais de Ética para a utilização de animais de laboratório.

Os animais foram anestesiados por meio da injeção intraperitoneal (i.p.), com solução de cetamina (Ketamina Agener $^{\circledR}$ a $10 \%$ ), na dose de $0,1 \mathrm{ml}$ por $100 \mathrm{~g}$ de peso corpóreo dos ratos, xilazina (Anesedan ${ }^{\circledR}$ a $2 \%$ ), na dose de $0,07 \mathrm{ml}$ por $100 \mathrm{~g}$ de peso e fentanil (Fentanest ${ }^{\circledR}$ a $5 \%$ ) na dose de $0,001 \mathrm{ml}$ por $100 \mathrm{~g}$ de peso. Em todos os animais foi administrada no pré-operatório 
dose única de Pentabiótico Veterinário (benzilpenicilina benzatina) 600.000 U, benzilpenicilina procaína 300.000UI, benzilpenicilina potássica 300.000UI, sulfato de dihidroestreptomicina $250 \mathrm{mg}$ e sulfato de estreptomicina 250mg (Pentabiótico Veterinário Pequeno Porte $^{\circledR}$ ) por via intramuscular, na dose de $0,1 \mathrm{ml}$ para cada $100 \mathrm{~g}$ de peso corpóreo do rato.

O modelo experimental proposto no estudo consistiu na retirada do núcleo pulposo (NP) da cauda do animal, colocação sobre diferentes estruturas na região lombar (dura-máter, gânglio da raiz dorsal e raiz nervosa) e avaliação dos parâmetros indicativos de hiperalgesia mecânica e térmica. $\mathrm{O}$ estudo foi realizado em quatro etapas: na 1a, o NP foi colocado sobre o gânglio da raiz dorsal (GRD), sobre a dura-máter ou sobre o nervo espinal, seguida da avaliação da intensidade da hiperalgesia, com o objetivo de determinar qual estrutura é mais sensível ao contato com os elementos do NP; na 2åa, o NP foi colocado sobre o GRD em três diferentes níveis da coluna lombar (L4, L5, L6), para verificar qual nervo seria mais suscetível às alterações induzidas pelo contato com o NP; na 3a a a hiperalgesia da pata traseira induzida pelo contato do NP foi comparada com a sensibilização induzida pelo contato de um tecido inerte (tecido gorduroso); e na $4^{\mathrm{a}}$, confirmação da ausência de comprometimento neurológico no modelo experimental e validá-lo.

A retirada do núcleo pulposo (NP) do disco intervertebral foi realizada na região sacrococcígea (base da cauda do rato) por meio de incisão na linha média da região de transição entre a quarta vértebra sacral e a primeira vértebra coccígea. $\mathrm{O}$ disco intervertebral foi bilateralmente exposto e, por meio de incisão transversal sobre o anel fibroso, o NP foi retirado. O material gelatinoso do NP foi coletado, pesado em balança de precisão (peso de 4 a $5 \mu \mathrm{g}$ em média) e, em seguida, depositado no local determinado para o estudo (duramáter, GRD ou raiz nervosa). Após o procedimento, a ferida cirúrgica foi suturada em um único plano, abrangendo a fáscia muscular e a pele (figura 1).
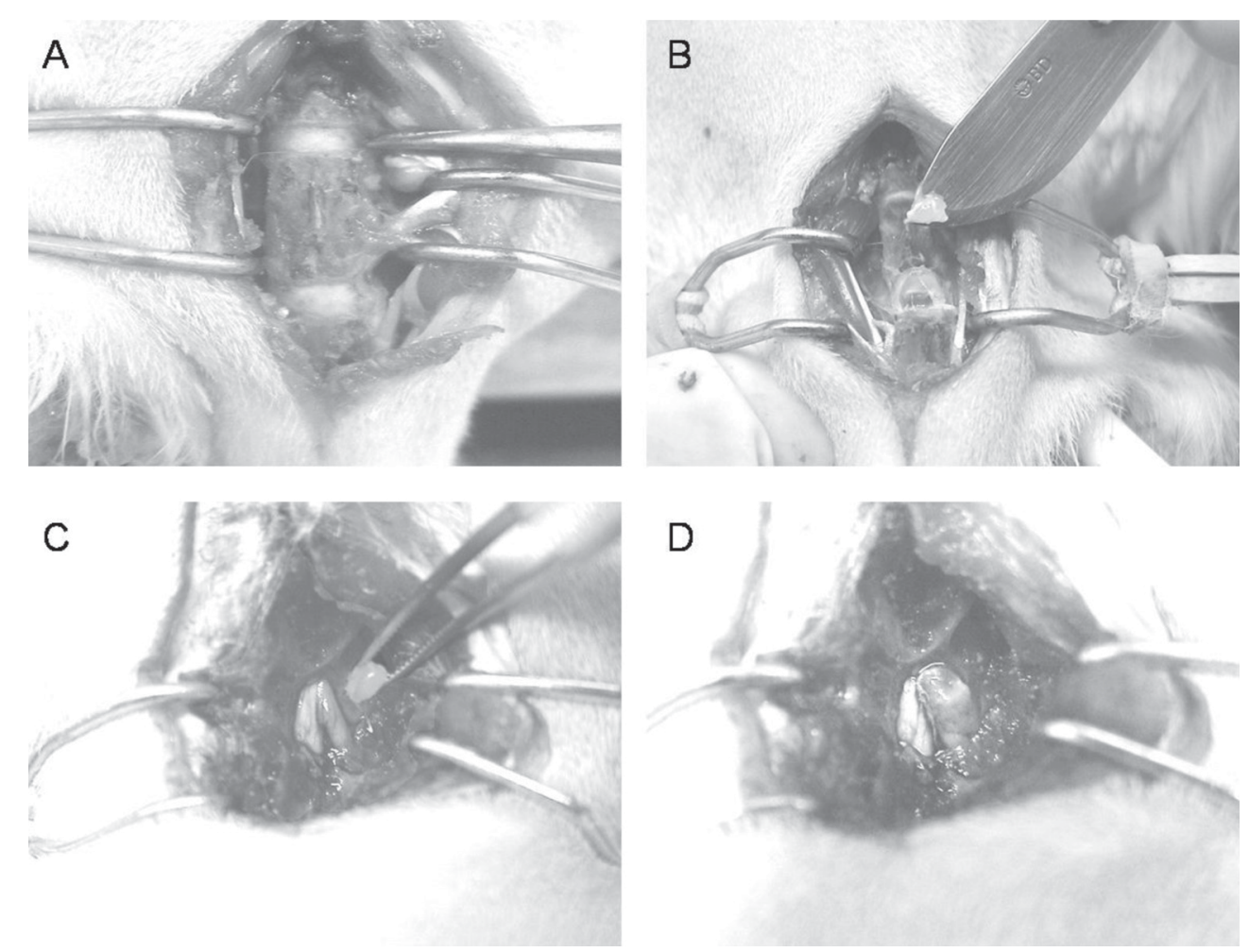

Figura 1 - Exposição do corpo e discos intervertebrais da região sacrococcígea (A) e remoção do núcleo pulposo (NP) (B); exposição do GRD da região lombar da coluna vertebral (C) e deposição do fragmento do NP sobre o GRD (D). 
A cirurgia para a colocação do conteúdo do NP sobre os tecidos nervosos foi realizada com o auxílio de lente ótica, com magnificação do campo de observação de 10 vezes. Por meio de laminectomia parcial hemilateral à direita e retirada do processo transverso vertebral no nível estabelecido, as estruturas (duramáter, GRD ou raiz nervosa) eram expostas e o conteúdo do NP, depositado (figura 1).

Nos animais do grupo-controle (falso-operados), os procedimentos de retirada do NP e reprodução de hérnia de disco foram realizados da mesma forma, porém o NP não foi depositado sobre as estruturas nervosas. Dessa maneira, as condições cirúrgicas experimentais entre os grupos foram mantidas.

A avaliação da hiperalgesia foi realizada por meio da observação dos reflexos motores e sensitivos, sendo a avaliação motora feita pelo teste de "rota-rod" e, a sensitiva, pelos testes de hiperalgesia mecânica (teste de pressão crescente da pata - von Frey eletrônico) e térmica (teste plantar de Hargreaves). Os parâmetros foram medidos por um avaliador independente que não possuía conhecimento do tipo de procedimento cirúrgico realizado nos animais.

TESTE DE PRESSÃo CRESCENTE NA PATA DE RATOS (VON FREY ELETRÔNICO) - A hiperalgesia mecânica foi avaliada por meio do teste de pressão crescente na pata de ratos, denominado de teste von Frey eletrônico (tenocicepção) $)^{(11)}$. O método consiste no uso de um anestesiômetro eletrônico (modelo 1601C, Life Science Instruments ${ }^{\circledR}$ ), o qual possui um transdutor de força conectado a um contador digital de força expressa em gramas (g). A precisão do aparelho é de $0,1 \mathrm{~g}$ e o mesmo é calibrado para registrar força máxima de $150 \mathrm{~g}$, mantendo a precisão de $0,1 \mathrm{~g}$ até a força de $80 \mathrm{~g}$. O contato do transdutor de força com a pata é realizado por meio de uma ponteira descartável de polipropileno com $0,5 \mathrm{~mm}$ de diâmetro adaptada ao transdutor. Os animais são colocados em caixas de acrílico, medindo 12 x 20 x $17 \mathrm{~cm}$, cujo assoalho consiste de uma rede de malha igual a $5 \mathrm{~mm}^{2}$, constituída de arame não maleável de $1 \mathrm{~mm}$ de espessura, durante 15 minutos antes do experimento, para adaptação ao ambiente. Espelhos posicionados $25 \mathrm{~cm}$ abaixo das caixas de ex- perimentação facilitam a visualização das plantas das patas dos animais. $\mathrm{O}$ experimentador aplica, por entre as malhas da rede, força linearmente crescente no centro da planta da pata do rato até que o animal produza uma resposta de retirada e "sacudida" da pata estimulada. Os estímulos são repetidos até seis vezes, em geral até que o animal apresente três medidas similares com uma nítida resposta de "sacudida" após a retirada da pata. A intensidade da hiperalgesia é então quantificada como sendo a variação na força ( $\Delta$ de reação em gramas) obtida subtraindo-se a média dos três valores expressos em gramas (força) observada antes do procedimento experimental (zero hora ou estado basal) da média de três valores em gramas (força) após a administração de estímulos hiperalgésicos ou, no presente caso, a realização dos procedimentos cirúrgicos, nos tempos determinados pelos protocolos definidos.

TESTE DE HIPERALGESIA TÉRMICA DE HARGREAVES (PLANTAR TEST) - A avaliação da hiperalgesia térmica foi realizada por meio do teste plantar de Hargreaves et al ${ }^{(10)}$. Esse teste consiste no aquecimento, por meio de fonte de luz infravermelha dirigida, da região plantar da pata traseira do rato, até que o animal apresente o comportamento da retirada da pata ou o aquecimento da pata atinja o limite de tempo predeterminado (tempo de corte de 12 segundos).

Os animais são colocados em compartimentos de acrílico individual e posicionados sobre uma superfície de vidro especial, que permite a passagem de forma homogênea da luz e do calor, durante cinco minutos, para adaptação ao ambiente. Após esse período, uma fonte de luz infravermelha, colocada sob cada uma das patas traseiras do animal, é acionada juntamente com um cronômetro eletrônico, até que o animal retire a pata, quando, então, a fonte de luz e o relógio param automaticamente.

São realizadas três medidas com intervalo de tempo de 5 a 10 min. As medidas de latência de retirada da pata são então expressas como avaliações percentuais relativas às patas-controle (contralaterais) em cada tempo de observação. Na situação em que o animal não apresenta reação, é estipulado um tempo limite máxi- 
mo de corte do experimento de 12 segundos para a preservação da sua integridade física.

TESTE DE ROTA-ROD - A avaliação motora foi realizada por meio do teste de rota-rod, metodologia descrita por Kuribara et $a l^{(12)}$. O aparelho de rota-rod consiste basicamente de um cilindro distante $7 \mathrm{~cm}$ da superfície da mesa, que gira a velocidade constante movido por um motor e regulado para o modo de velocidade constante de $18 \mathrm{rpm}$. Para manter-se sobre cilindro giratório, o animal necessita locomover-se, de modo que a capacidade locomotora pode ser então quantificada cronometrando-se o tempo de permanência do rato sobre o cilindro. Durante o estudo, os animais foram avaliados no dia anterior ao das cirurgias, para que ocorresse habituação e aprendizado, e em dias posteriores, para avaliação da função motora.

ETAPAS EXPERIMENTAIS - 1 a etapa - Essa etapa foi realizada para estudar a reação das estruturas nervosas (raiz nervosa, dura-máter e GRD) quando em contato com o NP. Foram utilizados 20 animais divididos em quatro grupos experimentais, sendo cada grupo experimental formado por cinco animais $(\mathrm{N}=5)$. Todos os grupos foram submetidos ao tratamento cirúrgico, sendo o NP depositado em diferentes estruturas nervosas: raiz nervosa, dura-máter ou GRD. No grupo controle, foi realizada apenas a abordagem cirúrgica, sem deposição do NP. Os animais foram avaliados pelos métodos de von Frey eletrônico e Hargreaves, três, sete, $14,21,28,36$, 42 e 49 dias após o procedimento cirúrgico. 2a etapa - Esses experimentos foram realizados para estabelecer qual raiz nervosa (L4, L5, L6) seria mais sensível aos métodos de avaliação de hiperalgesia utilizados. Vinte animais foram divididos em quatro grupos experimentais, sendo cada grupo formado por cinco animais. Todos os grupos foram submetidos ao tratamento cirúrgico; o conteúdo do NP foi colocado sobre os GRDs em diferentes níveis da coluna lombar do animal (L4, L5 e L6). No grupo controle foi realizada a abordagem cirúrgica das estruturas, porém sem a deposição do NP. Os animais foram avaliados pelos métodos de von Frey eletrônico e Hargreaves, três, sete, 14, 21, 28, 36, 42 e 49 dias após o procedimento cirúrgico. 3a etapa - Após a determinação da estrutura ner- vosa que apresentava maior sensibilidade quando em contato com o NP, foi realizado um experimento complementar para avaliar se as mudanças no limiar hiperalgésico eram resultado da reação inflamatória causada pelo procedimento cirúrgico ou do contato com os componentes do NP. Foram utilizados 20 animais, divididos em dois grupos experimentais formados por 10 animais. Ambos os grupos foram submetidos ao tratamento cirúrgico; em um dos grupos, foi depositado o conteúdo do NP sobre o GRD-L5 e, no outro grupo, foi realizado o procedimento cirúrgico e depositado tecido gorduroso sobre o GRD. Os animais foram avaliados pelos métodos de von Frey eletrônico e Hargreaves três, sete, 14, 21, 28, 36, 42 e 49 dias após o procedimento cirúrgico. 4a etapa - Esta etapa foi realizada para confirmar a ausência de lesão motora no modelo cirúrgico utilizado. Assim, após os procedimentos cirúrgicos, foi feita avaliação motora dos animais no teste de rota-rod. Foram utilizados 20 animais, divididos em quatro grupos experimentais com cinco animais em cada grupo. Os grupos foram formados de acordo com o tipo de procedimento realizado: grupo I - animais submetidos à cirurgia para reprodução da hérnia de disco, com o conteúdo do NP sendo depositado sobre o GRD-L5; grupo II - cirurgia para reprodução da hérnia de disco, com lesão mecânica por esmagamento do GRD-L5; grupo III - grupo controle, animais normais e não operados; grupo IV - grupo controle operado, submetido ao procedimento cirúrgico para reprodução da hérnia de disco, porém sem a deposição do conteúdo do NP. Os animais foram avaliados por meio do teste de avaliação motora (rotarod) três, sete e 14 dias após o procedimento cirúrgico.

AnÁlise estatísticA - Os resultados foram expressos como média e erro padrão da média (EPM) de pelo menos cinco animais por grupo (com exceção do experimento 4 , , no qual a resposta induzida pelo contato com o núcleo pulposo foi comparada com a reação induzida pelo tecido gorduroso. Nesse experimento foram utilizados 10 animais). A análise das curvas obtidas foi feita por análise de variância ANOVA oneway, seguida por teste de Bonferroni para as compara- 
ções múltiplas, ou teste $t$ de Student, para dados nãopareados, tendo sido considerados como significativos os valores de $\mathrm{p}<0,05$.

\section{RESULTADOS}

Os resultados serão apresentados de acordo com as etapas realizadas.

Os dados correspondentes à $1^{\underline{a}}$ etapa, realizada para identificação da estrutura nervosa mais sensível ao contato com o NP, estão representados no gráfico 1. Foi verificado que tanto a hiperalgesia mecânica quanto a térmica, provocadas pelo contato com o NP com o GRD, apresentaram maior intensidade quando comparadas com as induzidas pelo contato do NP com as demais estruturas nervosas, em todos os períodos de observação (gráfico 1).

Imediatamente após os procedimentos cirúrgicos, um período inicial de maior intensidade de sensibilidade da pata dos animais foi observado nos dois métodos comportamentais, período que foi declinando até o último dia de medida (49o dia). Entretanto, mesmo com essa alteração na hiperalgesia da pata traseira, os animais que tiveram o NP colocado sobre o GRD foram os que apresentaram maior sensibilização, enquanto os outros grupos não diferiram estatisticamente entre si.

A

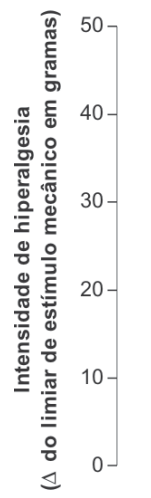

von Frey eletrônico

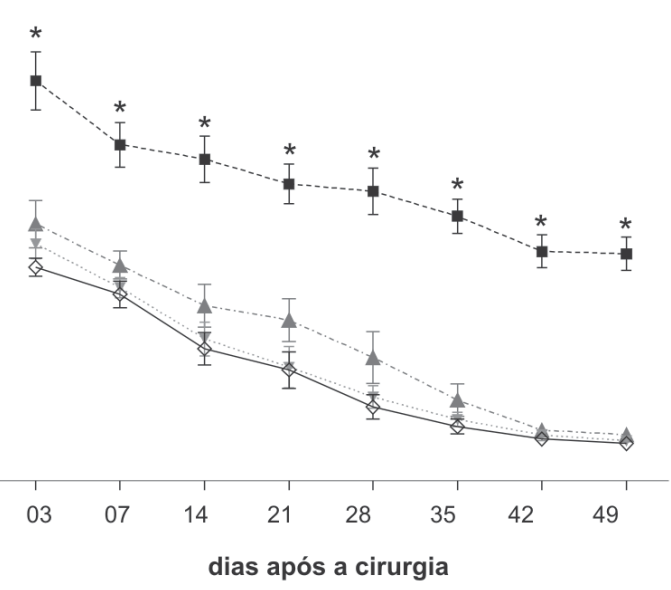

Grupos experimentais:
Com relação à 2a etapa, realizada para determinar qual nível lombar é mais sensível aos métodos de avaliação de hiperalgesia utilizados, os dados obtidos estão representados no gráfico 2 .

A intensidade da hiperalgesia térmica (gráfico 2) no grupo de animais em que o nível L5 (GRD-L5) foi estimulado apresentou comportamento distinto em relação aos demais grupos (GRD-L4, GRD-L6 e grupo controle) ao longo do período de tempo de observação. Além disso, a hiperalgesia térmica nos grupos GRD-L4, GRD-L6 e controle parece não ter diferido estatisticamente durante o período de observação. Mais ainda, não foi verificada redução acentuada da hiperalgesia térmica a partir do 14ํ dia de avaliação no grupo GRD-L5.

Em relação à avaliação da sensibilidade à estimulação mecânica, a diferença na hiperalgesia mecânica (gráfico 2) apresentada pelos grupos GRD-L4 e GRD-L6 não foi significativa. Contudo, diferiu estatisticamente do grupo controle e do grupo GRD-L5, o qual apresentou hiperalgesia mais intensa.

Os resultados obtidos na 3 a etapa estão representados no gráfico 3. A avaliação da hiperalgesia por meio dos dois diferentes métodos mostrou diferença entre o grupo no qual o NP foi colocado sobre a raiz (grupo NP) e o grupo no qual foi depositado tecido gorduroso. No gru-

B

Hargreaves

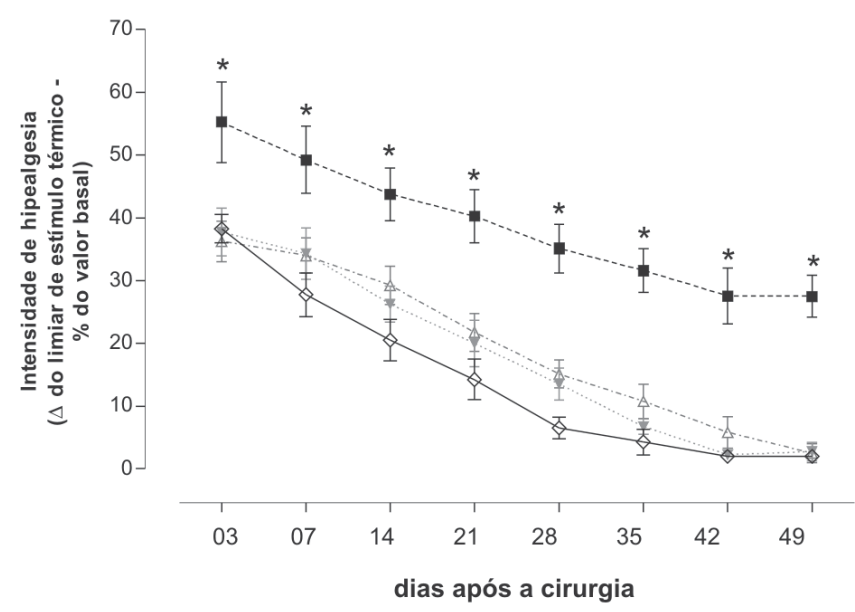

Gráfico 1 - Representação da variação na intensidade de hiperalgesia, avaliada pelos métodos de von Frey eletrônico (painel A) e Hargreaves (painel B), durante sete semanas (49 dias) após a deposição do núcleo pulposo sobre o gânglio da raiz dorsal (GRD) ( $\square$ ), sobre a dura-máter $(\Delta)$ ou sobre a raiz nervosa $(\boldsymbol{\nabla})$. Os resultados estão expressos como a média \pm EPM de cinco animais. ${ }^{*} \mathrm{p}<0,05$ quando comparados com o grupo-controle falso-operado $(\diamond)$ (ANOVA seguida do teste de Bonferroni). 
po NP, foi observada pequena redução na intensidade da hiperalgesia (tanto mecânica quanto térmica) ao longo do período, enquanto que no grupo de animais que tiveram tecido gorduroso depositado sobre a raiz nervosa essa redução foi menos intensa (gráfico 3).

A

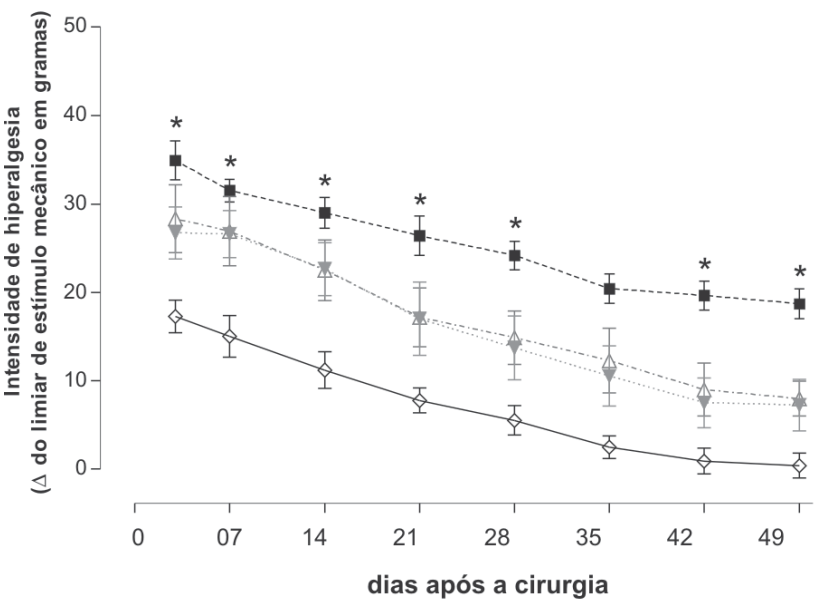

Grupos experimentais:

--- - - NP sobre o GRD 5

$\cdots \triangle$... NP sobre o GRD 4

B

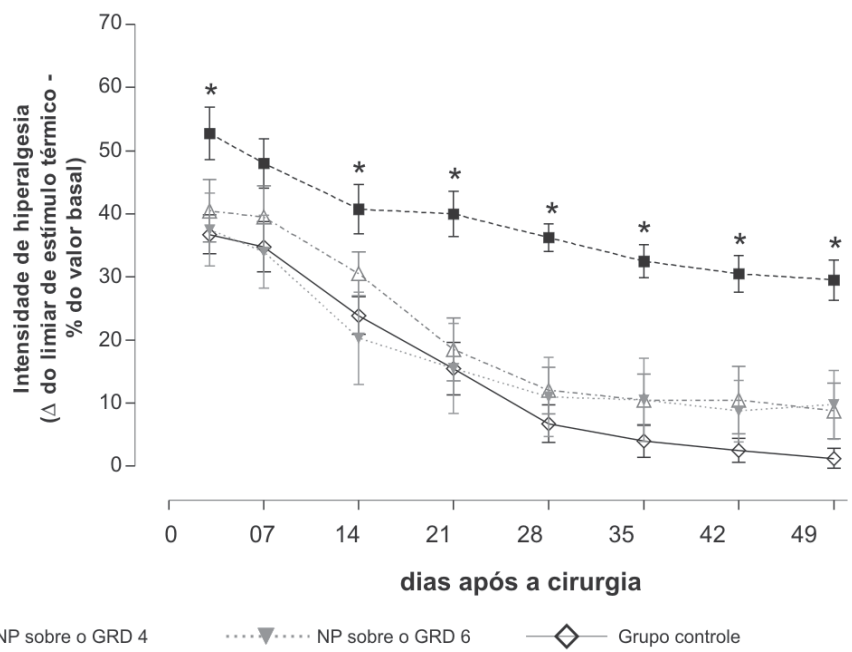

Gráfico 2 - Representação da variação na intensidade de hiperalgesia, avaliada pelos métodos de von Frey eletrônico (painel A) e Hargreaves (painel B), durante sete semanas (49 dias) após a deposição do núcleo pulposo sobre o gânglio da raiz dorsal (GRD) L4 $(\Delta)$, L5 ( $\square)$ ou L6 $(\boldsymbol{\nabla})$. Os resultados estão expressos como a média \pm EPM de cinco animais. ${ }^{*} \mathrm{p}<0,05$ quando comparados com o grupo-controle falso-operado $(\diamond)$ (ANOVA seguida do teste de Bonferroni).

A

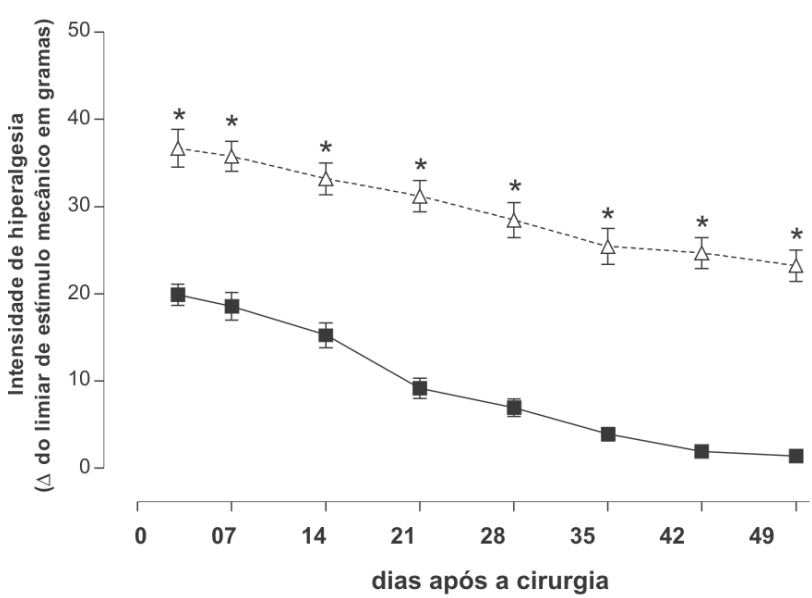

B

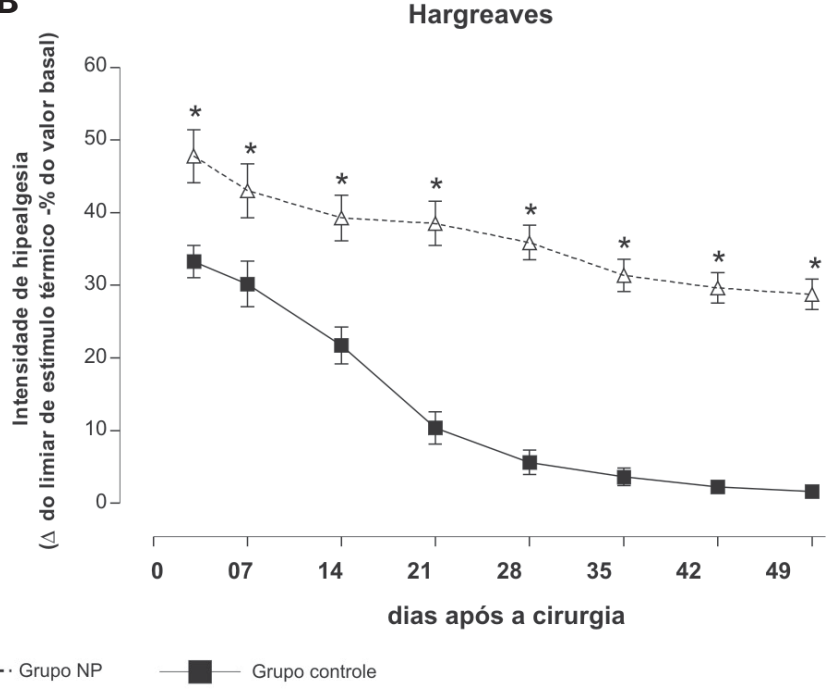

Gráfico 3 - Representação da comparação da intensidade de hiperalgesia induzida pela colocação do núcleo pulposo (grupo NP - $\Delta$-) ou de tecido gorduroso (grupo controle - -) sobre o gânglio da raiz dorsal L5, avaliada pelos métodos de von Frey eletrônico (painel A) e Hargreaves (painel B), durante sete semanas (49 dias). Os resultados estão expressos como a média \pm EPM de 10 animais. ${ }^{*} p<$ 0,05 quando comparados com o grupo controle (घ) (teste $t$ de Student para dados não pareados). 


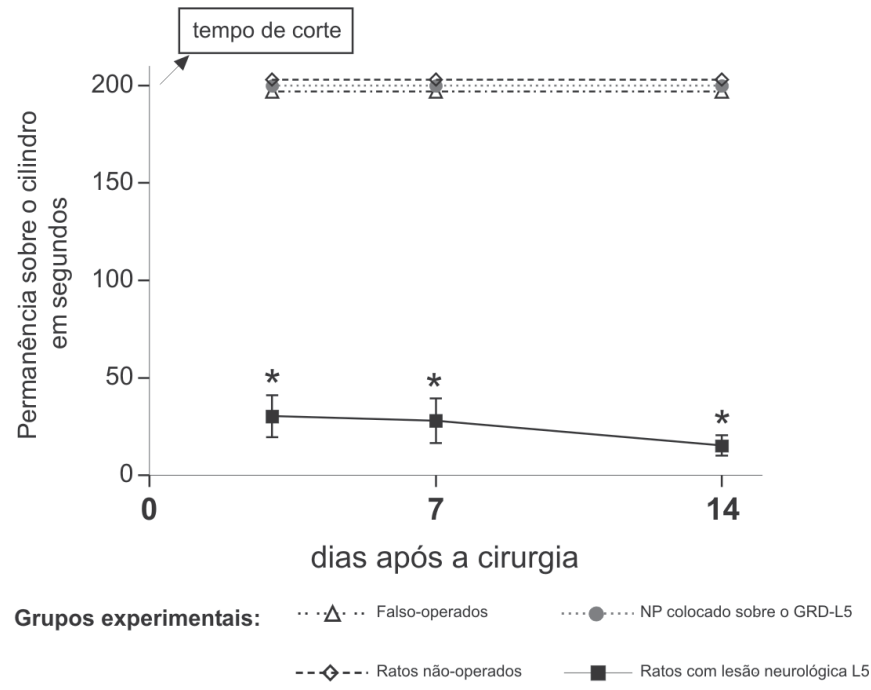

Gráfico 4 - Representação da avaliação da capacidade locomotora dos animais submetidos à cirurgia de indução de hérnia de disco lombar no teste de rota-rod. Foram comparados animais falso-operados $(\Delta)$, ratos que tiveram a hérnia induzida no GRDL5 $(\bullet)$, ratos normais $(\diamond)$ e ratos com lesão na medula espinal (घ). Os resultados estão expressos como a média \pm EPM de cinco animais por grupo. ${ }^{*} \mathrm{p}<0,05$ quando comparados com os grupos falso-operados $(\Delta)$, NP colocado sobre o GRD-L5 $(\bullet)$ e ratos não-operados $(\vartheta)$ (ANOVA seguida de teste de Bonferroni).

utilizados durante o estudo. Mais ainda, não foi observada diferença no padrão de atividade motora entre os grupos nos quais a lesão do tecido nervoso não foi realizada; esse padrão permaneceu constante durante todo o período de observação. Entretanto, o grupo cujo GRD foi lesionado apresentou discreta piora no decorrer do período (gráfico 4).

\section{DISCUSSÃO}

A compressão mecânica das raízes nervosas e as suas alterações decorrentes da atividade biológica ou bioquímica dos componentes do núcleo pulposo (NP) do disco intervertebral em contato com a raiz nervosa são os mecanismos específicos da gênese dos sintomas radiculares ${ }^{(4)}$. A compressão mecânica da raiz nervosa foi o mecanismo inicialmente reconhecido na época da descrição da doença ${ }^{(1)}$, enquanto que a atividade biológica do NP foi reconhecida muito tempo depois ${ }^{(8)}$.

A participação do NP na gênese dos sintomas da hérnia de disco foi experimentalmente demonstrada por Olmarker et al, em $1993^{(8)}$. A partir desses estudos, o potencial lesivo dos componentes do núcleo pulposo por meio da sua ação biológica ficou estabelecido, inaugurando, então, uma nova fase no âmbito do tratamento e da pesquisa desse tema. Inúmeros estudos experimentais têm sido realizados com o objetivo de elucidar quais são os mecanismos básicos envolvidos nesse fenômeno ${ }^{(9,13-15)}$. A incisão cirúrgica do ânulo fibroso de cães com a saída de pequena quantidade do NP foi suficiente para produzir alterações da estrutura e na função motora das raízes nervosas adjacentes ${ }^{(7)}$. Além disso, foi observado que a aplicação epidural do NP autólogo induziu edema intraneural e diminuição do fluxo sanguíneo do nervo ${ }^{(16)}$, além de alterações histológicas e redução da condução nervosa. Quando localizado fora do espaço intervertebral, o NP induz reação tecidual e apresenta potencial inflamatório com resposta leucotáxica ${ }^{(3,6)}$.

Observações clínicas como a dor de maior intensidade nas hérnias de disco não contidas e teste de elevação do membro inferior mais intenso ${ }^{(3,17)}$, no comportamento relacionado à dor associada à quantidade de NP no espaço epidural ${ }^{(4,17)}$, e ausência de dor durante o toque dos nervos que não estão em contato com o material herniado do NP confirmam as observações do potencial biológico do NP na gênese da dor na hérnia de $\operatorname{disco}^{(3-4,17)}$.

A importância do tema no âmbito de nossa área de atuação e a perspectiva da realização de estudos experimentais que poderão contribuir para o aprimoramento dos conhecimentos e tratamento da hérnia de disco motivaram o desenvolvimento de um modelo experimental.

Dessa forma, o modelo experimental proposto em nosso trabalho está relacionado com os fenômenos de hiperalgesia induzidos pela hérnia de disco que são ocasionados pelo contato do NP com a raiz nervosa, porém, as alterações sensitivas e motoras não foram consideradas no estudo.

O modelo desenvolvido confirmou as observações da produção das alterações na raiz nervosa pelo contato com o NP relatadas em estudos prévios ${ }^{(5-6,14,18-19)} \mathrm{e}$ foi possível identificar o gânglio da raiz dorsal (GRD) como sendo a estrutura que produziu as alterações mais intensas na sensibilidade a estímulos nocivos. Dessa maneira, nosso trabalho vem somar-se aos inúmeros 
estudos que focalizam a importância do GRD não apenas como o centro metabólico dos neurônios sensoriais aferentes primários, mas também como local de processamento de estímulos provenientes tanto dos tecidos periféricos como centrais ${ }^{(20-21)}$. Mais ainda, sua localização entre os compartimentos central e periférico, possibilitando receber estímulos provindos de diferentes tecidos, sugere que pode ter importância maior ainda que a que antes era dada no que se refere à detecção de estímulos patológicos ${ }^{(20,22-24)}$.

Apesar de a hiperalgesia ter sido observada quando o estímulo inflamatório (NP) era depositado em todos os níveis estudados da coluna lombar, o quinto nível lombar (GRD-L5) apresentou maior sensibilidade durante a avaliação da hiperalgesia, tanto mecânica quanto térmica. Esse fato corrobora os dados da literatura que mostram maior participação de fibras derivadas do GRD de L5 na inervação sensorial da região plantar da pata traseira dos ratos ${ }^{(25)}$, local no qual os estímulos de avaliação nociceptiva foram aplicados.

A realização do teste motor confirmou a ausência de lesão motora da raiz nervosa em contato com o NP, que poderia ter sido produzida durante a realização do procedimento cirúrgico e interferido nos resultados. A ausência da lesão motora confirma e evidencia a natureza biológica das alterações da raiz nervosa produzidas pelo contato com o NP.

As possíveis influências do processo inflamatório decorrente da realização do procedimento cirúrgico e sua influência sobre a raiz nervosa foram também eliminadas na elaboração do modelo experimental. A colocação do tecido gorduroso (material inerte) sobre a raiz nervosa e a realização do procedimento cirúrgico sem a colocação do NP sobre as estruturas nervosas destacaram o papel do NP na alteração da sensibilidade à dor.

Dessa maneira, o modelo proposto apresentou as vantagens econômicas da manipulação de animais de pequeno porte, permitiu a avaliação da hiperalgesia produzida pelo contato do NP com as estruturas nervosas por testes comportamentais bastante conhecidos e, em adição, realizar essa avaliação durante um período de tempo prolongado, já que a hiperalgesia se manteve por várias semanas.
Esse modelo experimental também permitiu a determinação do GRD como a estrutura mais sensível ao contato com o NP, uma vez que a deposição do estímulo (NP) sobre esse tecido produziu a hiperalgesia de maior intensidade. Ainda, o nível lombar cuja deposição do NP possibilitou melhor detecção da hiperalgesia (quinto nível da coluna lombar - GRD-L5) pelos métodos de avaliação na pata utilizados (testes de hiperalgesia mecânica e térmica) também pôde ser determinado.

Considerando que a dor é o sintoma mais difícil de ser avaliado em estudos experimentais, quando comparada com as alterações motoras ou sensitivas, a associação do protocolo cirúrgico proposto com testes validados de avaliação de sensibilidade nociceptiva torna o método aqui apresentado bastante útil no estudo de alterações sensitivas induzidas pelo extravasamento do NP e seu contato com as raízes nervosas. Cabe ainda comentar que o modelo possibilita o estudo de diferentes tipos de hiperalgesia induzidos pela hérnia de disco lombar, enquanto o método de von Frey eletrônico, como descrito por Vivancos et al ${ }^{(26)}$, avalia a sensibilidade à estimulação de origem mecânica e o método de Hargreaves et $a l^{(10)}$ focaliza a sensibilidade a estímulos térmicos de alta intensidade. As diferenças observadas entre as duas respostas nos sugerem que as fibras nervosas que detectam os estímulos nocivos se comportam de maneira diferente.

O modelo proposto e experimentalmente validado utilizando animais de pequeno porte apresenta vantagens econômicas e de manuseio, e permitiu identificar a estrutura e o nível da coluna espinhal mais sensível ao contato com o NP para o estudo da resposta hiperalgésica induzida pela hérnia de disco, a partir da estimulação de tecidos periféricos do animal estudado (pata), possibilitando sua utilização em estudos futuros.

\section{CONCLUSÕES}

1) A colocação do núcleo pulposo sobre a dura-máter, raiz nervosa ou gânglio da raiz dorsal da região lombar da coluna vertebral produz hiperalgesia na pata traseira de animais, sem a produção de alterações motoras.

2) Maior intensidade de hiperalgesia é produzida pelo contato do núcleo pulposo com o gânglio da raiz dorsal. 
3) O nível L5 apresenta maior sensibilidade para a avaliação da hiperalgesia por meio dos métodos de von Frey eletrônico e Hargreaves.

\section{AGRADECIMENTOS}

Os autores agradecem aos técnicos Ieda Regina dos Santos Schivo e Sérgio Roberto Rosa, pelo suporte durante os experimentos. Às agências de fomento à pesquisa: Fundação de Amparo à Pesquisa de São Paulo (FAPESP), Conselho Nacional de Desenvolvimento Científico e Tecnológico (CNPq) e Coordenação de Aperfeiçoamento de Pessoa de Nível Superior (CAPES).

\section{REFERENCIAS}

1. Mixter WJ. Rupture of the lumbar intervertebral disk: an etiologic factor for so-called "sciatic" pain. Ann Surg. 1937; 106(4):777-87.

2. Omarker K, Myers RR. Pathogenesis of sciatic pain: role of herniated nucleus pulposus and deformation of spinal nerve root and dorsal root ganglion. Pain. 1998;78(2):99-105.

3. Jönsson B, Strömqvist B. Clinical appearance of contained and noncontained lumbar disc herniation. J Spinal Disord. 1996; $9(1): 32-8$.

4. Olmarker K, Storkson R, Berge OG. Pathogenesis of sciatic pain: a study of spontaneous behavior in rats exposed to experimental disc herniation. Spine. 2002; 27(12):1312-7.

5. Arai I, Mao GP, Otani K, Konns S, Kikuchi S, Olmarker K. Indomethacin blocks the nucleus pulposus-induced effects on nerve root function. An experimental study in dogs with assessment of nerve conduction and blood flow following experimental disc herniation. Eur Spine J. 2004;13(8):691-4.

6. Kawakami M, Tamaki T, Matsumoto T, Kuribayashi K, Takenaka T, Shinozaki M. Role of leukocytes in radicular pain secondary to herniated nucleus pulposus. Clin Orthop Relat Res. 2000;(376):268-77.

7. Kayama S, Konno S, Olmarker K, Yabuki S, Kikuchi S. Incision of the anulus fibrosus induces nerve root morphologic, vascular, and functional changes. An experimental study. Spine. 1996;21(22):2539-43.

8. Olmarker K, Rydevik B, Nordborg C. Autologous nucleus pulposus induces neurophysiologic and histologic changes in porcine cauda equina nerve roots. Spine. 1993;18(11):1425-32. Comment in: Spine. 1994;19(20):2369-70.

9. Otani K, Arai I, Mao GP, Konno S, Olmarker K, Kikuchi S. Experimental disc herniation: evaluation of the natural course. Spine. 1997;22(24):2894-9.

10. Hargreaves K, Dubner R, Brown F, Flores C, Joris J. A new and sensitive method for measuring thermal nociception in cutaneous hyperalgesia. Pain. 1988;32(1):77-88.

11. Möller KA, Johansson B, Berge OG. Assessing mechanical allodynia in the rat paw with a new electronic algometer. $\mathbf{J}$ Neurosci Methods. 1998;84(1-2):41-7.
12. Kuribara H, Higuchi Y, Tadokoro S. Effects of central depressants on rota-rod and traction performances in mice. Jpn J Pharmacol. 1977;27(1):117-26.

13. Brisby H, Byröd G, Olmarker K, Miller VM, Aoki Y, Rydevik B. Nitric oxide as a mediator of nucleus pulposus-induced effects on spinal nerve roots. J Orthop Res. 2000;18(5):815-20.

14. Habtemariam A, Virri J, Grönblad M, Holm S, Kaigle A, Karaharju E. Inflammatory cells in full-thickness anulus injury in pigs. An experimental disc herniation animal model. Spine. 1998;23(5):524-9.

15. Olmarker K, Iwabuchi M, Larsson K, Rydevik B. Walking analysis of rats subjected to experimental disc herniation. Eur Spine J. 1998;7(5):394-9.

16. Yabuki S, Kikuchi S, Olmarker K, Myers RR. Acute effects of nucleus pulposus on blood flow and endoneurial fluid pressure in rat dorsal root ganglia. Spine. 1998;23(23):2517-23.

17. Nygaard OP, Mellgren SI, Osterud B. The inflammatory properties of contained and noncontained lumbar disc herniation. Spine. 1997;22(21):2484-8.

18. Murata Y, Nannmark U, Rydevik B, Takahashi K, Olmarker K. Nucleus pulposus-induced apoptosis in dorsal root ganglion following experimental disc herniation in rats. Spine. 2006; 31(4):382-90.

19. Olmarker K, Blomquist J, Strömberg J, Nannmark U, Thomsen P, Rydevik B. Inflammatogenic properties of nucleus pulposus. Spine. 1995;20(6):665-9.

20. Devor M. Unexplained peculiarities of the dorsal root ganglion. Pain. 1999;Suppl 6:S27-35.

21. Ferrari LF, Cunha FQ, Parada CA, Ferreira SH. A novel technique to perform direct intraganglionar injections in rats. $\mathrm{J}$ Neurosci Methods. 2007;159(2):236-43.

22. Ji RR, Samad TA, Jin SX, Schmoll R, Woolf CJ. p38 MAPK activation by NGF in primary sensory neurons after inflammation increases TRPV1 levels and maintains heat hyperalgesia. Neuron. 2002;36(1):57-68.

23. Michael GJ, Averill S, Nitkunan A, Rattray M, Bennett DL, Yan Q, Priestley JV. Nerve growth factor treatment increases brainderived neurotrophic factor selectively in TrkA-expressing dorsal root ganglion cells and in their central terminations within the spinal cord. J Neurosci. 1997;17(21):8476-90.

24. Porreca F, Lai J, Bian D, Wegert S, Ossipov MH, Eglen RM, et al. A comparison of the potential role of the tetrodotoxininsensitive sodium channels, PN3/SNS and NaN/SNS2, in rat models of chronic pain. Proc Natl Acad Sci U S A. 1999; 96(14):7640-4. Erratum in: Proc Natl Acad Sci U S A. 1999; 96(18):10548.

25. Takahashi Y, Nakajima Y. Dermatomes in the rat limbs as determined by antidromic stimulation of sensory C-fibers in spinal nerves. Pain. 1996;67(1):197-202.

26. Vivancos GG, Verri WA Jr, Cunha TM, Schivo IR, Parada CA, Cunha FQ, Ferreira SH. An electronic pressure-meter nociception paw test for rats. Braz J Med Biol Res. 2004;37(3): 391-9. 